\title{
Quelles médiations pour une gestion efficace de la transposition didactique en anglais de spécialité ?
}

\section{Marie-Christine Deyrich}

\section{(2) OpenEdition}

\section{Journals}

Édition électronique

URL : http://journals.openedition.org/asp/1909

DOI : 10.4000/asp.1909

ISBN : 978-2-8218-0384-8

ISSN : 2108-6354

Éditeur

Groupe d'étude et de recherche en anglais de spécialité

Édition imprimée

Date de publication : 1 octobre 2001

Pagination : 143-152

ISSN : 1246-8185

Référence électronique

Marie-Christine Deyrich, « Quelles médiations pour une gestion efficace de la transposition didactique en anglais de spécialité ? », ASp [En ligne], 31-33 | 2001, mis en ligne le 12 novembre 2010, consulté le 19 avril 2019. URL : http://journals.openedition.org/asp/1909; DOI : 10.4000/asp.1909

Ce document a été généré automatiquement le 19 avril 2019

Tous droits réservés 


\title{
Quelles médiations pour une gestion efficace de la transposition didactique en anglais de spécialité?
}

\author{
Marie-Christine Deyrich
}

1 Dans l'apprentissage de la langue vivante étrangère hors immersion, la médiation occupe une place privilégiée : à la jonction entre les stratégies d'enseignement et les stratégies d'apprentissage. Il est de ce fait intéressant de se demander comment, dans cette rencontre de deux logiques dissemblables, les stratégies d'enseignement peuvent avoir un impact aussi bénéfique que possible sur l'apprentissage. Pour proposer des éléments de réponse, nous nous attacherons plus particulièrement à la façon dont la médiation didactique peut accompagner l'apprenant dans la construction de son propre savoir sur la langue et jouer ainsi un rôle déterminant dans l'évolution favorable de son système de représentations métalinguistiques. Dès lors, l'interrogation porte sur les moyens dont nous disposons pour agir efficacement à ce niveau et concerne prioritairement la substance des contenus et les modalités de leur mise en forme pédagogique dans l'enseignement-apprentissage. Il s'agit donc d'examiner en fonction de quels critères l'intervention didactique sur les savoirs linguistiques pourra s'avérer utile à l'apprenant.

Dans cette visée, nous sollicitons le concept de transposition didactique. En effet, comme nous l'avons montré dans deux expérimentations menées auprès d'un public de spécialistes d'autres disciplines (Deyrich $2000: 259-502)^{1}$, pour être utile à l'apprentissage, le savoir linguistique est indispensable à une élaboration de contenus qui soient à la fois substantiels et rationnels mais, pour que les contenus soient appropriables par l'apprenant et réinvestis dans les quatre savoir-faire, ils ne peuvent en aucun cas se satisfaire d'une simple importation en provenance des domaines de référence; il est totalement impossible et absurde que le savoir soit livré à l'état brut. En d'autres termes, pour que la grammaire soit réellement opératoire, on devra déterminer la nature et la fonction des traitements auxquels il faudra soumettre ce savoir théorique pour qu'il joue un rôle effectif et favorable dans l'apprentissage. 
Se trouve ainsi posée la question des moyens d'action et des points de repère dont nous disposons pour que le processus transpositif facilite l'apprentissage. Dans cette perspective, la médiation didactique, globalement orientée vers un progrès, transforme et simplifie l'ensemble des données pour qu'elles deviennent appropriables et qu'elles soient réinvesties dans les quatre savoir-faire en langue. D'autre part, bien qu'elle implique un processus de simplification didactique, il est paradoxalement indispensable que la gestion de la transposition soit informée de la complexité du système. Par conséquent, l'adoption d'un «méta-point de vue » (Morin 1990), garant de la cohérence d'ensemble, est de mise pour guider la localisation et la définition de chacun des différents niveaux où des transformations doivent intervenir.

Un tel élargissement de l'angle d'analyse nous permet d'observer que l'intervention didactique se caractérise notamment par une démultiplication, au fil des différentes instanciations du savoir. C'est pourquoi, afin de mettre le concept de médiation en perspective avec le processus transpositif, nous estimons qu'il convient de parler de médiations au pluriel. Celles-ci constituent, selon nous, autant de possibilités d'intervention dont nous disposons pour viser l'efficacité. En conséquence, leur examen devrait nous permettre de dégager quelques critères pour opérer des choix didactiques motivés.

\section{Le savoir en position médiate : implication du concept de transposition didactique dans le domaine des langues vivantes étrangères}

5 Le savoir linguistique n'existe pas en tant que tel dans l'enseignement de la langue vivante étrangère (LVE). En effet, la grammaire ne présente un intérêt que si elle devient opératoire dans les savoir-faire langagiers. L'attribution de cette position médiate au savoir théorique doit forcément être prise en compte dans la planification didactique. C'est pourquoi il convient non seulement de déterminer quels aspects du concept de transposition didactique peuvent contribuer à une définition de contenus rationnels, mais aussi d'envisager les adaptations nécessaires, liées à la spécificité de notre domaine.

\subsection{La chaîne transpositive : de l'« objet de savoir » à l'« objet appris »}

6 Le concept de transposition didactique, qui met en évidence le " passage du savoir savant au savoir enseigné » (Chevallard [1985] 1991 : 20), implique qu'il y ait à la fois une relation entre deux objets de nature différente et une rupture, une distanciation produite par l'introduction dans la sphère didactique. Les transformations subies par le savoir se succèdent au fil d'un parcours pour lequel Chevallard (ibid. 39) propose le schéma 1.

Schéma 1

$\rightarrow$ objet de savoir $\rightarrow$ objet à enseigner $\rightarrow$ objet d'enseignement

7 Le savoir constitue la trame du parcours transpositif: l'« objet de savoir» (qui a été sélectionné dans le domaine des "savoirs savants») se métamorphose en "objet à enseigner » à l'issue d'une « acclimatation institutionnelle » (ibid. 15) - il devient apte à 
être enseigné -, puis en "objet d'enseignement " (savoirs préparés à être enseignés). Nous avons élargi le champ de ce schéma (Deyrich 2000 : 158) pour inclure aussi bien l'action du professeur que celle de l'élève (voir figure 1).

Figure 1. La médiation

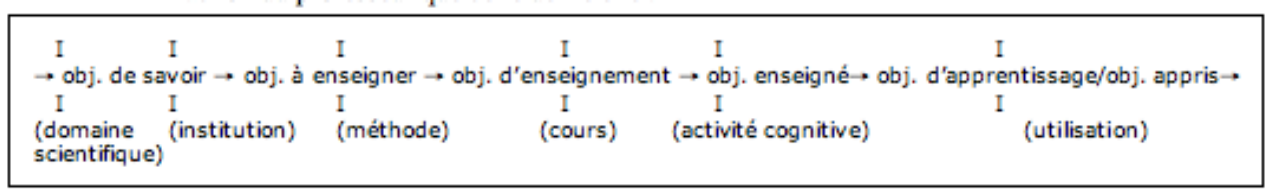

L'« objet d'enseignement » laisse place dans la salle de classe à l'« objet enseigné » qui devient alors "objet d'apprentissage » et se transforme enfin en "objet appris», donc susceptible de transfert. D'autre part, on observe que chaque croisement de la trame avec la chaîne transpositive actualise le savoir, générant ainsi un nouvel « objet » qui intègre les contraintes et les déterminations dans les transformations successives.

\subsection{L'« objet » d'enseignement-apprentissage de la LVE}

Le savoir linguistique sur lequel nous nous appuyons - la description linguistique de la langue - doit être à la fois fiable et utile à l'apprentissage. La théorie des opérations énonciatives d'Antoine Culioli présente ces caractéristiques, notamment par sa mise en relation constante de la forme et du sens (Gilbert 1993) et par l'ancrage de la réflexion au niveau des représentations métalinguistiques. L'apport potentiel pour l'apprentissage est considérable, puisque celui-ci intervient en profondeur, dans la mise en place de "schèmes conceptuels" (Vergnaud 1990), des représentations métalinguistiques des apprenants, pour ce qui concerne les langues vivantes étrangères.

Pour atteindre ce niveau, la transposition didactique implique des choix et des infléchissements des emprunts théoriques se fondant strictement sur leur utilité pour l'efficacité de l'apprentissage (Bailly 1997 : 30-31). Le savoir de la communauté « savante » ne peut intervenir en l'état dans la salle de classe : «linguistics should essentially be behind the teacher, not in front of the class » (Culioli 1979 : 209).

11 En conséquence, des transformations radicales doivent intervenir, depuis l'introduction d'éléments explicatifs du domaine des savoirs de référence jusqu'à la mise en œuvre d'explications qui soient adaptées aux besoins et intégrées dans des dispositifs concourant à l'acquisition de savoir-faire langagiers.

\subsection{Gestion du processus transpositif}

12 Dès lors que le point de vue est interventionniste ou, en d'autres termes, si l'on considère que chaque médiation est un élément clé dans la simplification didactique, l'étude du parcours transpositif a un but d'ordre praxéologique : en permettre la gestion. Or, il s'agit d'une tâche particulièrement délicate, à la jonction de deux logiques. D'une part, la gestion du processus transpositif doit tenir compte de la spécificité de l'apprentissage dans l'élaboration d'un « étayage » (Bruner 1983) et dans sa mise en œuvre lors de ce que Bruner qualifie d'« interaction de tutelle » (ibid. 277-278) et qui laisse une large place à la construction cognitive du Sujet-apprenant, facilitant ainsi l'émergence personnelle du sens (Laks 1996: 170) il s'agit, selon Bange (1999), de contribuer à l'élaboration d'un 
«étayage métacognitif ». D’autre part, nous estimons que, pour que l'« objet-enseigné » bénéficie de l'apport de la description linguistique de référence, il importe que la démarche de simplification didactique respecte une cohérence conceptuelle d'ensemble qui tienne compte de la spécificité de l'« objet-langue ».

\section{Médiations didactiques au fil du parcours du savoir}

\subsection{Les étapes de la simplification didactique}

Tentons à présent de suivre le parcours de la linguistique à la grammaire du point de vue des stratégies d'enseignement. Pour déterminer quand et dans quel but l'intervention didactique doit transformer et simplifier, on peut distinguer d'emblée trois étapes, comme l'illustre la figure 2 (d'après Deyrich $2000: 218$ ).

Figure 2

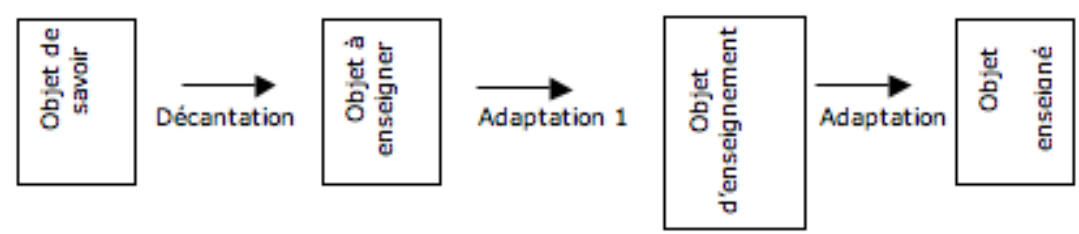
réalisation morpho-syntaxique pour adopter un point de vue métalinguistique. L'adaptation 1 met en perspective les besoins des apprenants et les explications susceptibles d'agir sur leurs représentations métalinguistiques. L'adaptation 2 intervient au cours de la séance d'enseignement-apprentissage, dans un " étayage » (correspondant aux procédures de guidage et au métalangage) qui suit pas à pas le cheminement de l'apprentissage. On observe que chacune de ces étapes imprime un écart qui confirme la différence de nature et de fonction de l'« objet ». Il ne faut cependant pas perdre de vue que la maitrise de chacun des écarts doit être assurée pour préserver la cohérence d'ensemble.

\subsection{Les médiations dans l'organisation générale de la transposition didactique}

Pour examiner de façon plus précise les modalités d'intervention dont nous disposons, il est utile de les situer dans le parcours global du savoir. Il convient alors d'abandonner le mode de représentation linéaire pour rendre compte de la complexité du système, notamment en différenciant les stratégies d'enseignement et les stratégies d'apprentissage, tout en en situant les points de convergence.

Cette mise en perspective montre que les trois étapes précédemment délimitées demeurent pertinentes à deux conditions. Il faut qu'elles soient encadrées par une évaluation (état des lieux puis bilan) qui leur donne une orientation conforme aux besoins et qui les intègre dans une spirale ascendante. De plus, pour que les modalités d'intervention soient clairement identifiables pour chacune de ces étapes, il convient de les démultiplier pour dégager des étapes intermédiaires. 
Dans le schéma de la figure 3 (d'après Deyrich 2000 : 543), nous donnons, sur un axe chronologique, un aperçu des formes que devraient revêtir les différentes médiations, en interrelation avec le rôle qu'elles jouent pour chacun des maillons successifs de la chaîne transpositive.

Figure 3

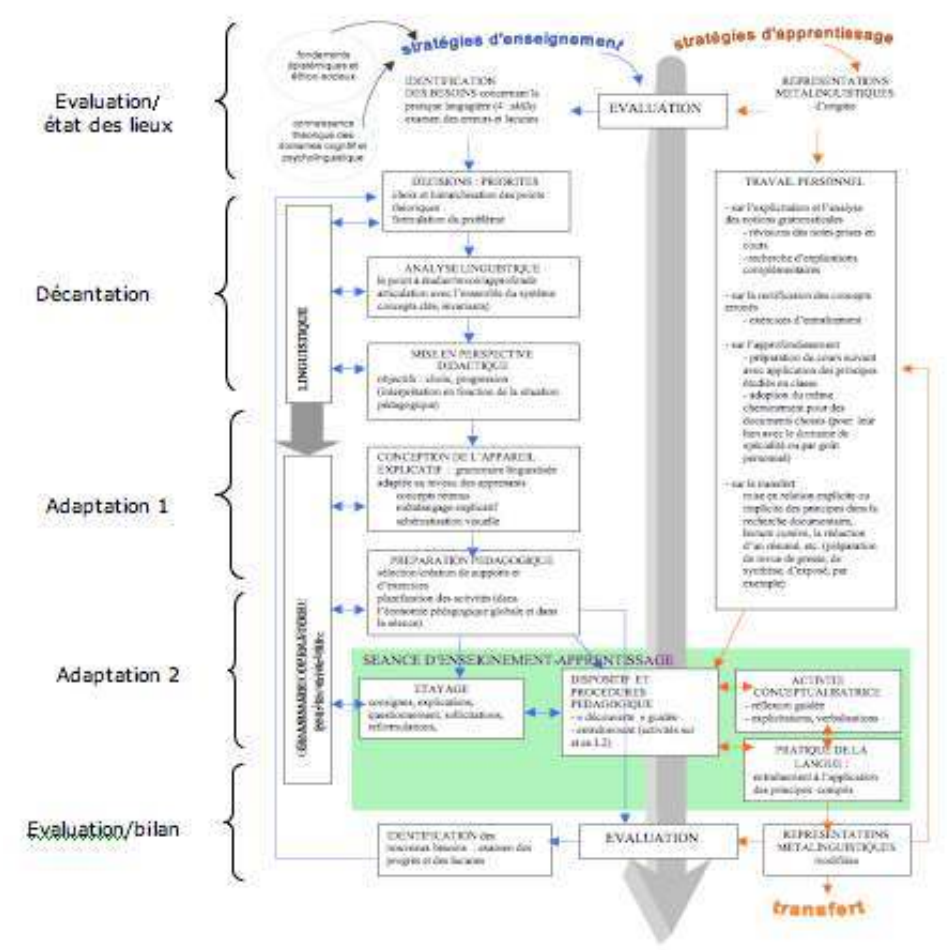

\subsection{Contrôle des écarts : développer une stratégie globale et des micro-stratégies}

Sur ce que Morin appelle "paradigme de la complexité » (1990: 77-104), l'approche transpositive suppose, en fonction d'un plan donné qui est l'émanation des besoins observés, l'adoption d'une vision d'ensemble favorisant l'établissement de liens (pour mettre en relation les connaissances nouvelles et les acquis antérieurs, fixer des points de repère, établir des correspondances entre les opérations langagières de même nature, etc.).

Dans la structure globale (cf. figure 3), l'attention se porte plus particulièrement sur les articulations stratégiques -celles qui permettent d'assurer le contrôle des écarts pour canaliser le parcours du savoir dans le but que l'objet d'enseignement-apprentissage soit : transmissible (simplifications des concepts et du métalangage de l'appareil explicatif) et conceptualisable, c'est-à-dire servant de tremplin à l'activité métalinguistique (transformations en exercices et activités).

Bien que l'interrelation entre ces deux objectifs complémentaires ne fasse aucun doute, leur différenciation permet de caractériser, d'une part, les étapes qui concernent plus particulièrement la définition de contenus et, d'autre part, celles qui mettent l'accent sur les modalités pédagogiques de la mise en œuvre. 
Enfin, s'agissant des niveaux d'intervention sur les savoirs, la démultiplication des médiations nous amène à distinguer deux phases stratégiquement distinctes malgré leur interdépendance :

- dans un premier temps, le contrôle porte sur les ponts qui sont construits à partir de la linguistique (formulation du problème, analyse linguistique puis mise en perspective didactique),

- ensuite, le contact avec la linguistique disparaît complètement au profit d'une grammaire linguistisée opératoire pour les savoir-faire, sur laquelle se fonde la « mise en scène » (Chevallard [1985] 1991) du savoir (conception de l'appareil explicatif, préparation pédagogique, étayage).

\section{Le parcours transpositif revisité : le cas de l'anglais de spécialité à l'université}

\subsection{Pertinence de l'approche transpositive dans l'enseignement de la LVE de spécialité}

Nos étudiants, spécialistes d'autres disciplines que l'anglais, sont des apprenants avancés. De ce fait, médiation rime bien souvent avec remédiation: les premières médiations n'ayant pas abouti, notre rôle est alors de faciliter la mise en place d'un apprentissage sur d'autres bases. Nous en déduisons que, dans ce cadre, le remède peut aisément accorder une large part à la réflexion métalinguistique pour agir sur les représentations des apprenants. Une telle démarche est d'autant plus réalisable qu'elle intervient dans un contexte où l'ancrage dans la spécialité du domaine étudié représente un véritable atout. En se fondant sur des documents liés à la spécialité étudiée, les activités communicatives associées à une approche conceptualisatrice des savoirs sur la langue octroient à l'apprentissage une dimension inhabituelle voire novatrice qui encourage les étudiants à exercer plus volontiers leur métier d'apprenant dans la mesure où ils en situent l'enjeu et acceptent le « partage de la tâche » (Perrin 1999 : 8).

Pour répondre à cette attente, l'enseignant de langue de spécialité est fréquemment amené à inventer tout (depuis la définition d'objectifs jusque la mise en œuvre dans la salle de classe) ou partie des dispositifs d'enseignement-apprentissage, en prenant appui sur des documents propres à la spécialité. Ceux-ci symbolisent en quelque sorte la matérialisation d'un domaine partagé, propice à la construction de l'apprentissage en situation. La préparation du cours est de ce fait essentielle; le temps d'amont se trouve particulièrement valorisé (Perrin 1995). Nous en déduisons que, dans la mesure où l'enseignant de spécialité doit nécessairement transformer, adapter, simplifier les éléments dont il dispose, le support d'une théorisation de la pratique qu'offre le concept de transposition didactique peut contribuer à une élaboration de contenus rationnels et à une définition des modalités de présentation aux étudiants qui tiennent compte de leurs itinéraires d'apprentissage. 


\subsection{Impact de la spécificité de l'enseignement de la LVE sur la transposition}

la conception élargie de la prise en charge du processus transpositif par l'enseignant de spécialité, on relève, parmi les aspects les plus significatifs, que le travail prépédagogique, sur lequel les stratégies d'enseignement se fondent, comporte notamment :

- un travail de prospection dans le domaine de spécialité qui requiert que des lignes de conduite (limites, orientations, priorités) soient fixées par les soins de l'enseignant, et qui suppose une recherche documentaire approfondie associée à un recours à des références spécialisées (dictionnaires, banques de données, etc.) ;

- des contacts et une mise en commun allant si possible jusqu'au travail d'équipe (avec les collègues spécialistes, avec les enseignants de la spécialité, dans les sous-groupes de spécialité) ;

- la fréquentation de la recherche de la spécialité, qu'elle soit directe (dans les contacts avec des chercheurs, l'assistance à des colloques, la lecture d'actes et d'articles) ou indirecte (dans les traductions et les conseils pour les collègues spécialistes, par exemple);

- une remise en question et des évolutions constantes : les nombreux apports transdisciplinaires, qu'il s'agisse de la recherche, de l'actualité ou encore des technologies de l'information et de la communication, doivent enrichir les connaissances concernant l'articulation du domaine de spécialité et des pratiques langagières.

En conséquence, la réflexion sur la transposition doit élargir le champ d'investigation pour tenter d'incorporer les manifestations didactiques des apports innovants ci-dessus mentionnés. De ce point de vue, on remarque, en premier lieu, la présence indispensable des ressources documentaires du domaine de spécialité, et, d'autre part, l'intégration de plus en plus fréquemment nécessaire des technologies de l'information et de la communication. Pour examiner comment le cheminement transpositif s'en trouve infléchi, voire modifié, nous nous limiterons ici au premier de ces traits caractéristiques.

\subsection{Complexification du parcours transpositif dans la LVE de spécialité : le cas de l'intégration des ressources documentaires}

Les ressources documentaires correspondent pour nous à l'ensemble des éléments (quels qu'ils soient) dont nous pouvons disposer dans la spécialité, c'est-à-dire les documents prélevés pour servir de base à la didactisation. Leur position est centrale dans la définition de contenus car elles influent presque systématiquement sur les décisions et les priorités. On peut en déduire que, dans l'enseignement de la LVE de spécialité, la transposition n'est pas déclenchée exclusivement par les problèmes relevés dans l'évaluation car, en règle générale, elle découle de la conjonction des centres d'intérêt de spécialité (ressources documentaires) et linguistiques (problèmes et lacunes repérés dans les quatre savoir-faire).

La présence très marquée des documents provenant du domaine de spécialité ajoute un point de départ à la structure de base et nous amène à estimer qu'une complexification intervient à l'origine du parcours. Le phénomène se manifeste par un dédoublement de l'entrée dans le processus transpositif. Cette démultiplication suppose que les médiations prennent en compte des besoins d'apprentissage de deux ordres : ceux dont l'évaluation nous a permis de dresser un état des lieux des représentations métalinguistiques et ceux 
établis de manière prospective à partir du prélèvement opéré dans les ressources documentaires, en amont de la didactisation.

Cependant, la complexification ne vient en rien obérer la suite du cheminement transpositif. En effet, les étapes qui suivent le point d'entrée doivent être parcourues les unes après les autres : il est impossible que des supports -quelle que soit leur qualité intrinsèque- puissent intervenir directement, sans aucune analyse préalable. Tout « raccourci » irait à l'encontre de l'approche transpositive dont nous avons montré l'intérêt pour la formation.

Nous reprenons dans la figure 4 la partie concernée de la figure 3 pour observer l'impact des ressources documentaires sur la transposition.

Figure 4

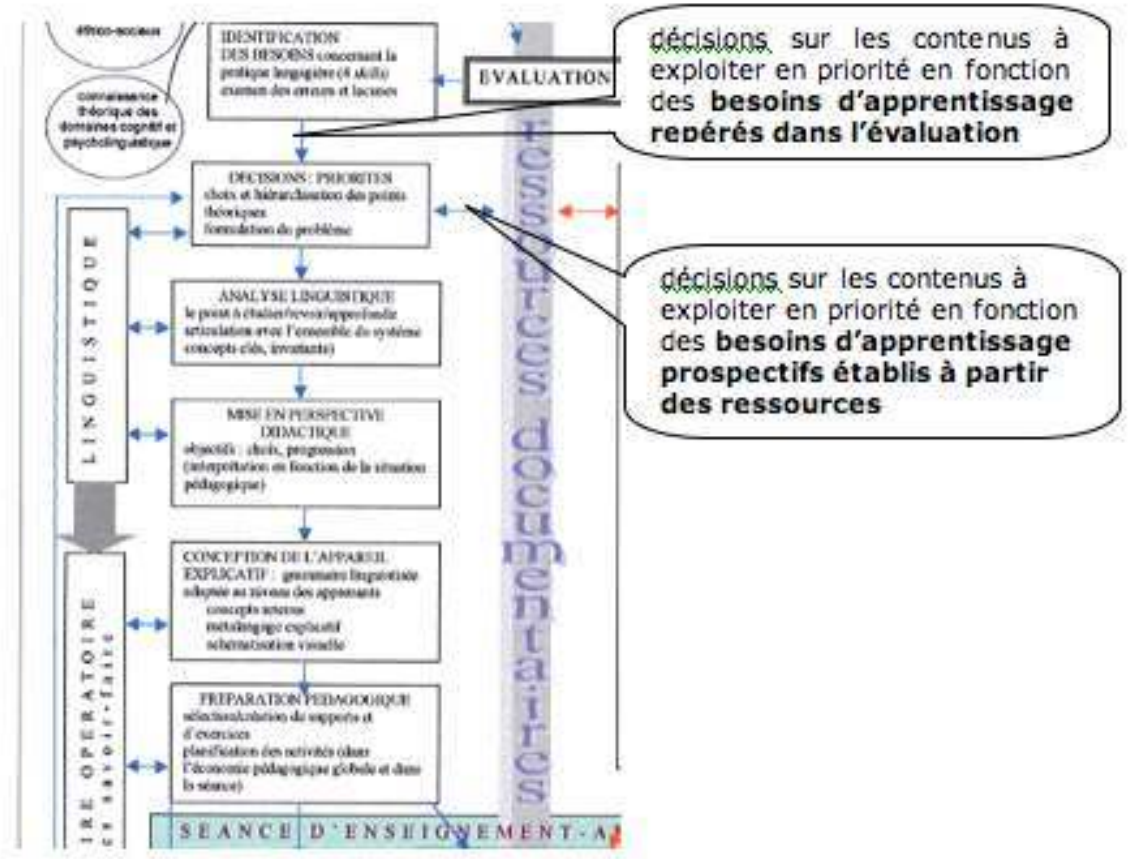

30 À la suite du double point d'entrée pour la prise de décisions, il apparaitt souhaitable que les médiations soient conformes à la logique transpositive décrite précédemment: une première série de transformations où le contact est maintenu avec la linguistique puis la préparation pédagogique au cours de laquelle les transformations prennent appui sur une grammaire opératoire pour les savoir-faire.

\section{Conclusion}

31 L'enseignement de spécialité ne peut, selon nous, faire l'économie d'une transposition de la linguistique à une grammaire opératoire dans les savoir-faire en langue. Une position inverse serait d'autant plus regrettable que la diversité des sources de spécialité (et donc des didactisations) offre une grande richesse énonciative et des possibilités d'exploitation intéressantes.

Il est important que la médiation didactique intervienne de manière suffisamment informée dans le parcours du savoir afin que celui-ci joue un rôle déterminant dans 
l'apprentissage. Pour que l'enseignant de spécialité dispose des moyens pour mettre en place une transposition opérante, il est utile qu'il puisse repérer et analyser chacune des articulations stratégiques, lorsque l'intégration dans la situation d'enseignementapprentissage d'éléments propres au domaine de spécialité sera source d'enrichissement du parcours du savoir et facilitera l'apprentissage.

De ce point de vue, l'enseignant de spécialité a la possibilité d'être un "transposeur" professionnel prenant en charge la totalité du processus, l'ensemble des médiations. Sa maitrise du processus transpositif inclut notamment l'exploitation d'apports du domaine de spécialité qui viennent enrichir l'organisation générale et lui donnent des moyens supplémentaires pour viser l'efficacité.

Enfin, le cadre général de la transposition didactique doit être aménagé pour rendre compte de la spécificité de la médiation didactique dans l'enseignement-apprentissage de l'anglais de spécialité dans l'enseignement supérieur, s'agissant en particulier de la présence nécessaire des ressources documentaires. Cependant, les étapes successives du parcours transpositif ont tout lieu d'être suivies pas à pas pour tenir compte de la complexité de l'ensemble du parcours.

\section{BIBLIOGRAPHIE}

Bailly, Danielle. 1997. Didactique de l'anglais. Objectifs et contenus de l'enseignement, vol. $\mathrm{n}^{\circ} 1$. Paris : Nathan.

Bange, Pierre. 1999. «L'usage de la règle dans l'enseignement et l'apprentissage de langue étrangère $"$, Congrès FOCAL.

Bruner, Jérôme. 1987 [1983]. Le Développement de l'enfant. Savoir faire, savoir dire. Paris : Presses universitaires de France.

Chevallard, Yves. 1991 [1985]. La Transposition didactique, du savoir savant au savoir enseigné. Grenoble : La Pensée sauvage.

Culioli, Antoine. 1979. " Why teach how to learn to teach what is best learnt untaught ». Cahiers de Charles V 1, 199-210.

Deyrich, Marie-Christine. 2000. « La transposition didactique dans l'anglais de spécialité à l'université. De la linguistique à une grammaire opératoire pour la maîtrise des savoir-faire en langue étrangère ». Thèse de doctorat, Université Paris VII Denis Diderot.

Gilbert, Eric. 1993. « La théorie des opérations énonciatives d'Antoine Culioli ». In Cotte P. et al., Les théories de la grammaire anglaise en France. Paris : Hachette, 63-96.

Laks, Bernard. 1996. Langage et cognition, l'approche connexionniste. Paris : Hermès.

Morin, Edgar. 1990. Introduction à la pensée complexe. Paris : E.S.F.

Perrin, Michel. 1995. «Les langues de spécialité, facteur de progrès pédagogique ». In Budin G. (dir.), Proceedings of the $10^{\text {th }}$ European LSP Symposium, Vienne (Autriche), 47-83. 
Perrin, Michel. 1999. « Le plus vieux métier du monde n'est pas celui qu'on croit ». La Tribune Internationale des Langues Vivantes 25, 5-15.

Vergnaud, Gérard. 1990. «La Théorie des champs conceptuels ». Recherches en didactique des mathématiques 10/23, 133-170.

\section{NOTES}

1. Le présent article se situe donc dans le prolongement de la recherche effectuée dans le cadre de notre thèse (Deyrich 2000). Deux expérimentations, l'une portant sur la détermination nominale, l'autre sur l'expression de la modalité épistémique, ont montré l'impact très favorable sur l'apprentissage d'une transposition didactique prenant appui sur une analyse préalable des acquis et des besoins pour l'élaboration d'un appareil explicatif visant le niveau des représentations métalinguistiques.

\section{RÉSUMÉS}

La question de la médiation sera abordée à partir du concept de transposition didactique dans le but de déterminer comment l'intervention enseignante peut simplifier les savoirs sur la langue pour qu'ils deviennent, chez les apprenants, opératoires dans la pratique de la langue, contribuant ainsi aux progrès de l'apprentissage. Afin de définir des critères pour une jonction favorable entre les stratégies d'enseignement et les stratégies d'apprentissage, nous nous concentrerons sur les différentes étapes du parcours transpositif de la linguistique à la grammaire, depuis la recherche d'explications dans les théories de référence jusqu'à la mise en œuvre dans la classe de langue. Enfin, nous tenterons de caractériser les aménagements nécessaires pour que le cadre général ainsi établi prenne en compte la spécificité de l'enseignement-apprentissage de l'anglais de spécialité dans l'enseignement supérieur.

The mediation issue will be addressed in the context of teaching applications in order to determine how teacher intervention can simplify knowledge about language so that, for learners, it becomes operational in language usage and thus contributes to learning progress. In order to establish criteria for a harmonised relationship between teaching and learning strategies, we shall focus on the different stages of the transposition from linguistics to grammar, from explanatory research in classificatory theory to its implementation in language classes. Finally, we shall investigate how this general framework has to be adapted to take account of the particular type of mediation required in the teaching and learning of English for specific purposes within higher education.

\section{INDEX}

Mots-clés : grammaire, progrès (de l'apprentissage), simplification, stratégie d'enseignement, transposition didactique

Keywords : didactic transposition, grammar, progress (learning), strategy (teaching) 


\section{AUTEUR}

\section{MARIE-CHRISTINE DEYRICH}

Marie-Christine Deyrich, après avoir été PRAG d'anglais à l'Université Charles de Gaulle Lille 3 auprès d'un public de spécialistes d'autres disciplines, a été nommée maître de conférences à l'IUFM de l'académie de Montpellier à la rentrée universitaire 2001. Sa recherche porte sur la didactique de l'anglais.mc.deyrich@wanadoo.fr 